\title{
Quantum Incompressibility of a Falling Rydberg Atom, and a Gravitationally-Induced Charge Separation Effect in Superconducting Systems
}

\author{
R.Y. Chiao • S.J. Minter $\cdot$ K. Wegter-McNelly • \\ L.A. Martinez
}

Received: 7 March 2010 / Accepted: 21 December 2010 / Published online: 12 January 2011

(C) The Author(s) 2011. This article is published with open access at Springerlink.com

\begin{abstract}
Freely falling point-like objects converge toward the center of the Earth. Hence the gravitational field of the Earth is inhomogeneous, and possesses a tidal component. The free fall of an extended quantum mechanical object such as a hydrogen atom prepared in a high principal-quantum-number state, i.e. a circular Rydberg atom, is predicted to fall more slowly than a classical point-like object, when both objects are dropped from the same height above the Earth's surface. This indicates that, apart from transitions between quantum states, the atom exhibits a kind of quantum mechanical incompressibility during free fall in inhomogeneous, tidal gravitational fields like those of the Earth.

A superconducting ring-like system with a persistent current circulating around it behaves like the circular Rydberg atom during free fall. Like the electronic wavefunction of the freely falling atom, the Cooper-pair wavefunction is quantum mechanically incompressible. The ions in the lattice of the superconductor, however, are not incompressible, since they do not possess a globally coherent quantum phase. The resulting difference during free fall in the response of the nonlocalizable Cooper pairs of electrons and the localizable ions to inhomogeneous gravitational fields is predicted to lead to a charge separation effect, which in turn leads to a large Coulomb force that opposes the convergence caused by the tidal gravitational force on the superconducting system.
\end{abstract}

R.Y. Chiao ( $\square)$

Schools of Natural Sciences and Engineering, University of California, P.O. Box 2039,

Merced, CA 95344, USA

e-mail: rchiao@ucmerced.edu

S.J. Minter · L.A. Martinez

School of Natural Sciences, University of California, 5200 N. Lake Rd., Merced, CA 95343, USA

K. Wegter-McNelly

School of Theology, Boston University, 745 Commonwealth Ave., Boston, MA 02215, USA 
A "Cavendish-like" experiment is proposed for observing the charge separation effect induced by inhomogeneous gravitational fields in a superconducting circuit. The charge separation effect is determined to be limited by a pair-breaking process that occurs when low frequency gravitational perturbations are present.

Keywords Quantum mechanics · Rydberg atom · Gravity · Equivalence principle · Uncertainty principle

\section{Introduction}

Experiments at the frontiers of quantum mechanics and gravity are rare. We would like to explore in this essay, in honor of Danny Greenberger and Helmut Rauch, situations which could lead to such experiments. The key is to understand the phenomenon of "quantum incompressibility" of macroscopically coherent quantum matter in the presence of inhomogeneous, tidal gravitational fields, such as the Earth's. See Fig. 1.

As an example of "quantum incompressibility" during free fall of an extended quantum object, let us first consider the single electron of a circular Rydberg atom [1] (ignoring electron spin), which is prepared in the state

$$
\left|n, l=n-1, m_{l}=n-1\right\rangle,
$$

where $n$ is the principal quantum number, which is large, i.e., $n \gg 1$, and $l=n-1$ is the maximum possible orbital angular momentum quantum number for a given $n$, and $m_{l}=l=n-1$ is the maximum possible azimuthal quantum number for a given $l$, i.e., the "stretched" state. The $z$ axis has been chosen to be the local vertical axis located at the center of mass of the atom. Then the wavefunction of this electron in polar coordinates $(r, \theta, \phi)$ of the hydrogenic atom in this state is given by [2, p. 253]

$$
\Psi_{n, n-1, n-1}(r, \theta, \phi)=N_{n, n-1, n-1}\left(r \sin \theta e^{i \phi}\right)^{n-1} \exp \left(-\frac{r}{n a_{0}}\right),
$$

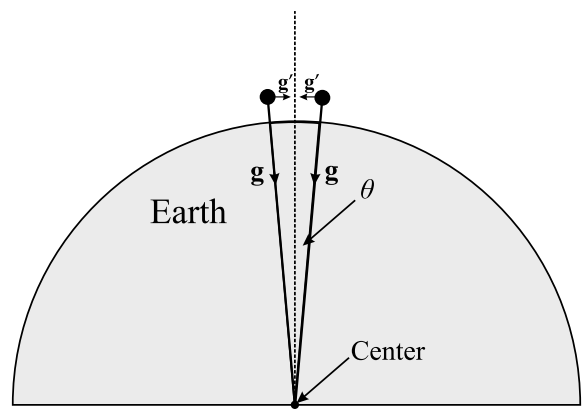

Fig. 1 Two nearby, freely falling, point-like objects dropped from the same height above the Earth's surface follow converging trajectories that are inclined at a slight angle $\theta$ with respect to the vertical plumb line equidistant between them. According to a distant inertial observer, the radial convergence of these objects' trajectories towards the center of the Earth causes them to undergo small horizontal components of acceleration $\mathbf{g}^{\prime}$ of the radial acceleration $\mathbf{g}$. These components are equivalent to a tidal gravitational force that, in a Newtonian picture, causes the two objects to converge toward one another 
Fig. 2 A circular Rydberg atom in the state $\mid n, l=n-1$, $\left.m_{l}=n-1\right\rangle$ has a strongly peaked, ring-like probability distribution, i.e., an "electron cloud," indicated by the heavy black loop. Currents in this state lead to a magnetic field $\mathbf{B}$, indicated by the directional loops. The $z$ axis is the local vertical axis

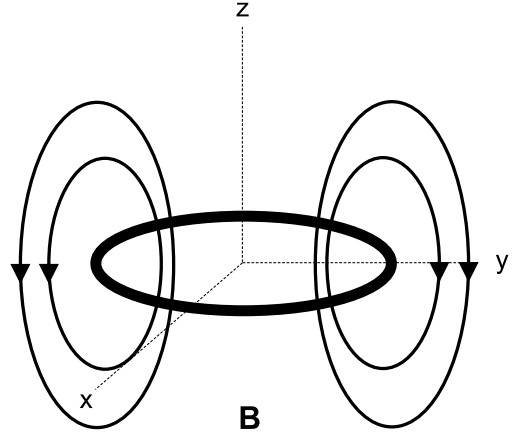

where $N_{n, n-1, n-1}$ is a normalization constant.

The probability density associated with this wavefunction has the form of a strongly peaked distribution which lies on the horizontal $(x, y)$ plane, in the shape of a ring of radius

$$
a_{n}=n^{2} \frac{\hbar^{2}}{m e^{2}}=n^{2} a_{0},
$$

where $a_{0}$ is the Bohr radius. Thus one recovers the Bohr model of the hydrogen atom in the correspondence-principle limit of large $n$. This ring-like probability distribution is illustrated in Fig. 2.

The question we would like to address here is this: How does the size of this atom change with time as it undergoes free fall in Earth's inhomogeneous, tidal gravitational field?

\section{An Analogy}

The magnetic moment of the Rydberg atom in the state (2) is quantized, and is given by

$$
\mu_{n}=n \frac{e \hbar}{2 m}=n \mu_{B},
$$

where $\mu_{B}$ is the Bohr magneton and $n$ is an integer.

The electron current density in the ring-like structure of a circular Rydberg atom in Fig. 2 is similar to that of a persistent supercurrent of Cooper pairs in a superconducting ring with a quantized flux given by

$$
\Phi_{n}=n \Phi_{0}=n \frac{h}{2 e},
$$

where $\Phi_{0}$ is the flux quantum and $n$ is an integer. The quantum incompressibility of the ring-like structure of a circular Rydberg atom, and the quantum incompressibility of the Cooper pairs of electrons in a superconducting ring, both arise from the same quantum mechanical principle, namely, the single-valuedness of the wavefunction 
after one round trip around the ring, which follows from the condition

$$
\oint_{\text {ring }} \nabla \varphi \cdot d \mathbf{l}=\Delta \varphi=2 \pi k,
$$

where $\varphi$ is the phase of the wavefunction, and $k$ is an integer corresponding to the state under consideration. Another necessary condition for quantum incompressibility is the existence of a substantial energy gap separating the $k$ th state from adjacent states of the system.

The analogy between the Rydberg atom and the superconducting ring is not a perfect one, since the selection rules for allowed transitions between adjacent states will be different in the two cases. The transitions $n \rightarrow n-1$ and $n \rightarrow n+1$ are electric-dipole allowed for the Rydberg atom, whereas the transitions $n \rightarrow n-1$ and $n \rightarrow n+1$ between adjacent flux-trapping states of the superconducting ring are highly forbidden. This is because a macroscopic number of identical Cooper pairs of electrons must all simultaneously jump from a state with $n \hbar$ units to a state with $(n-1) \hbar$ units or with $(n+1) \hbar$ units of angular momentum per electron pair. Hence the persistent current of a superconducting ring is highly metastable, and does not change with time, unless a macroscopic quantum transition occurs.

If the characteristic frequency of an external perturbation, such as that of the tidal gravitational fields acting on the system during free fall in Earth's gravity, is much less than the smallest energy gap of an allowed transition divided by Planck's constant, then the system cannot make a transition (i.e., a "quantum jump") out of its initial state. Thus it must stay rigidly in its initial state. (For a Rydberg atom with $n \simeq 100$, this transition frequency lies in the gigahertz range, so that this assumption is well satisfied.) The size of the circular Rydberg atom and the size of the persistent currents of the superconducting ring will therefore remain constant in time during perturbations arising from Earth's tidal fields during free fall, apart from a sequence of possible "quantum jumps" in a "quantum staircase," though such transitions occur only in highly unusual circumstances in the gravitational field of the Earth.

\section{The Quantum Incompressibility of the Rydberg Atom}

It has been previously shown [8] that the electron wavefunction of a hydrogen atom will be altered by the presence of curvature. The Hamiltonian operator for such an operator is given by

$$
H=H_{0}+H_{1},
$$

where $H_{0}$ is the unperturbed operator, and $H_{1}$ is an interaction Hamiltonian due to curvature. This interaction operator was shown to be

$$
H_{P} \equiv H_{1}=\frac{1}{2} m_{e} R_{0 l 0 m} x^{l} x^{m},
$$

where $m_{e}$ is the mass of the electron, $R_{0 l 0 m}$ are components of the Riemann curvature tensor, and $x^{l}$ and $x^{m}$ are components of the position operator of the electron. 
Equation (8) is obtained by taking the nonrelativistic limit of the Dirac equation embedded into curved spacetime $[9,10]$. The subscript $P$ is used for Parker, the author of the papers referenced here, and his index notation is adopted as well. Superscripts $l$ and $m$ are contravariant indices satisfying $l, m=1,2,3$, and should not be confused with quantum number $l$ or mass $m$. The index 0 refers to the time components. Greek indices are spacetime indices, and Latin indices are space indices only. The Einstein summation convention is used here as well, in which repeated indices are summed over all possible values.

Expressing these components of the Riemann tensor in terms of the gravitational scalar potential, which satisfies

$$
-\nabla \Phi=\mathbf{g}
$$

one can show that near the surface of the earth, the interaction Hamiltonian is given by

$$
H_{P}=\frac{m_{e} g}{2 R_{E}}\left(x^{2}+y^{2}-2 z^{2}\right)
$$

in Cartesian coordinates, or

$$
H_{P}=\frac{m_{e} g}{2 R_{E}}\left[r^{2}\left(3 \sin ^{2} \theta-2\right)\right]
$$

in spherical coordinates. It is worth noting that this interaction Hamiltonian is proportional to the second-degree, zero-order spherical harmonic $Y_{2}^{0}$. Thus, this interaction Hamiltonian represents a rank-2 angular momentum operator, since its angular dependence is quadrupolar [11]. One immediate consequence is that, by the WignerEckart theorem, there will be no effect on a hydrogen atom in a state with zero angular momentum (i.e. where $l=0$ ), to first order, through this interaction Hamiltonian.

The energy shift associated with $H_{P}$ is given by

$$
\Delta E_{P}=\frac{\left\langle\Psi\left|H_{P}\right| \Psi\right\rangle}{\langle\Psi \mid \Psi\rangle} .
$$

Taking the expectation values with respect to the stretched-state wavefunction in (2), one obtains

$$
\Delta E_{P} \approx \frac{m_{e} g}{2 R_{E}} a_{n}^{2}=\frac{m_{e} g a_{0}^{2}}{2 R_{E}} n^{4},
$$

where the approximation is valid for large values of principal quantum number $n$.

In addition to the energy shift derived from the interaction Hamiltonian $H_{P}$, one can use DeWitt's minimal coupling rule, which is associated with an asymptotically flat coordinate system, unlike the operator $H_{P}$, which is associated with the transverse-traceless gauge often used in general relativity. Let us show that quantum incompressibility is predicted to occur in a circular Rydberg atom, starting from DeWitt's minimal coupling rule. The DeWitt Hamiltonian for a freely falling hydrogenic atom, such as a circular Rydberg atom in presence of weak electromagnetic and gravitational fields, is given in SI units by

$$
H=\frac{1}{2 m}(\mathbf{p}-e \mathbf{A}-m \mathbf{h})^{2}+\frac{e^{2}}{4 \pi \varepsilon_{0} r},
$$


where $\mathbf{A}$ is the electromagnetic vector potential, and $\mathbf{h}$ is DeWitt's gravitational vector potential [3].

Before performing any quantitative analyses, it is stated here beforehand that while the Hamiltonian in (14) applies to the entire atom, one can apply a center-of-mass and relative coordinates transformation to the unperturbed Hamiltonian, treating the interaction Hamiltonians containing the vector potentials as perturbations. The fact that the proton has a much larger mass and is located at a much smaller distance from the center of mass causes the proton contributions to the energy shifts to be negligible compared to those of the electron contributions.

The interaction Hamiltonian for the A $\cdot \mathbf{A}$ term (the "Landau diamagnetism term" [4]) is given by ${ }^{1}$

$$
H_{\mathbf{A} \cdot \mathbf{A}}=\frac{e^{2}}{2 m_{e}} \mathbf{A} \cdot \mathbf{A} .
$$

In the symmetric gauge, where $\mathbf{A}=\frac{1}{2} \mathbf{B} \times \mathbf{r}=-\frac{1}{2} B\left(y \mathbf{e}_{x}-x \mathbf{e}_{y}\right)$, for $\mathbf{B}=B \mathbf{e}_{z}$, and where $\mathbf{e}_{x}, \mathbf{e}_{y}$, and $\mathbf{e}_{z}$ are the unit vectors along the $x, y$, and $z$ axes, respectively, this yields

$$
H_{\mathbf{A} \cdot \mathbf{A}}=\frac{e^{2} B^{2}}{8 m_{e}}\left(x^{2}+y^{2}\right),
$$

in Cartesian coordinates, or

$$
H_{\mathbf{A} \cdot \mathbf{A}}=\frac{e^{2} B^{2}}{8 m_{e}} r^{2} \sin ^{2} \theta
$$

in spherical coordinates, where $\theta$ is the azimuthal angle. The energy shift in firstorder perturbation theory resulting from the presence of the $\mathbf{A}$ field is given by

$$
\Delta E_{\mathbf{A} \cdot \mathbf{A}}=\frac{e^{2} B^{2}}{8 m_{e}}\left\langle\Psi_{n l m_{l}}\left|r^{2} \sin ^{2} \theta\right| \Psi_{n l m_{l}}\right\rangle .
$$

Recalling that the wavefunction for the circular Rydberg state is given by (2), the expectation value in (19) becomes

$$
\frac{\left\langle\Psi\left|r^{2} \sin ^{2} \theta\right| \Psi\right\rangle}{\langle\Psi \mid \Psi\rangle} \approx\left(n^{2} a_{0}\right)^{2}=a_{n}^{2}
$$

\footnotetext{
${ }^{1}$ In addition to the $\mathbf{A} \cdot \mathbf{A}$ and the $\mathbf{h} \cdot \mathbf{h}$ terms, there are also $\mathbf{A} \cdot \mathbf{p}$ and $\mathbf{h} \cdot \mathbf{p}$ terms in the perturbation Hamiltonian. Since the $\mathbf{A} \cdot \mathbf{A}$ term is quadratic in $\mathbf{A}$, and the $\mathbf{A} \cdot \mathbf{p}$ is linear, we must compute the $\mathbf{A} \cdot \mathbf{p}$ terms out to second order. The first-order $\mathbf{A} \cdot \mathbf{p}$ term gives rise to the linear Zeeman splitting effect,

$$
\Delta E_{\mathbf{A} \cdot \mathbf{p}}=\frac{\hbar e B}{2 m_{e}} m_{l},
$$

while the second-order term is zero due to the orthogonality of the atomic wavefunction states (since the wavefunctions are eigenstates of the $\mathbf{A} \cdot \mathbf{p}$ operator). The Hermicity of the $\mathbf{h}$ and $\mathbf{p}$ operators causes the $\{\mathbf{h}, \mathbf{p}\}$ terms to be zero in both first and second order. For details, see [5].
} 
for large values of the principal quantum number $n$, where $n \gg 1$. It follows that the first-order energy shift of the atom in the presence of a magnetic field is

$$
\Delta E_{\mathbf{A} \cdot \mathbf{A}} \approx \frac{e^{2} a_{n}^{2}}{8 m_{e}} B^{2} .
$$

This result implies that, in first-order perturbation theory, the size of the atom does not change in the presence of the applied DC magnetic field, in the sense that the root-mean-square transverse size of the atom, which is given by

$$
\left.a_{n}\right|_{\mathrm{rms}}=\sqrt{\left\langle\Psi\left|r^{2} \sin ^{2} \theta\right| \Psi\right\rangle}=a_{n}
$$

does not change with time, to first order, during the application of the DC magnetic field. Moreover, the wavefunction $\Psi_{n, n-1, n-1}$ remains unaltered in first-order perturbation theory in the presence of a weak applied field. Furthermore, this is still true for applied magnetic fields which vary sufficiently slowly in time, so that no transitions (i.e., "quantum jumps") can occur out of the initial state of the system $\Psi_{n, n-1, n-1}$. The concept of the "quantum incompressibility" of a Rydberg atom is thus a valid concept during the application of sufficiently weak, and sufficiently slowly varying, magnetic fields.

The energy shift given by (21) causes the atom to become a low-field seeker in inhomogeneous magnetic fields through the relationship

$$
\left(\mathbf{F}_{\mathbf{A} \cdot \mathbf{A}}\right)_{n}=-\nabla\left(\Delta E_{\mathbf{A} \cdot \mathbf{A}}\right)_{n} \approx-\frac{e^{2} a_{n}^{2}}{8 m_{e}} \nabla\left(B^{2}\right),
$$

where $\left(\mathbf{F}_{\mathbf{A} \cdot \mathbf{A}}\right)_{n}$ is the force on the atom in the ring-like state (2) in the presence of an inhomogeneous magnetic field.

Next, let us consider the more interesting case of when weak tidal gravitational fields are present without any accompanying electromagnetic fields, i.e., when $\mathbf{h} \neq \mathbf{0}$ and $\mathbf{A}=\mathbf{0}$. As before, the atom is initially prepared in the state given by (2) before it is released into free fall in the Earth's inhomogeneous gravitational field. The $z$ axis, which goes through the center of mass of the atom, is chosen to be the local vertical axis of the Earth's field. The horizontal tidal gravitational fields of the Earth experienced during free fall by the atom, as observed in the coordinate system of a distant inertial observer, where the $(x, y)$ plane is the local horizontal plane, will be given by

$$
\mathbf{h}(x, y, t)=\mathbf{v}(x, y, t)=\mathbf{g}^{\prime} t=\frac{g t}{R_{E}}\left(\mathbf{e}_{x} x+\mathbf{e}_{y} y\right),
$$

where $\mathbf{v}(x, y, t)$ is the velocity of a freely falling, point-like test particle located at $(x, y)$ and observed at time $t$ by the distant inertial observer [7], $\mathbf{g}^{\prime}$ is the horizontal component of Earth's gravitational acceleration arising from the radial convergence of free-fall trajectories towards the center of the Earth as seen by this observer (see Fig. 1), $R_{E}$ is the radius of the Earth, and $\mathbf{e}_{x}$ and $\mathbf{e}_{y}$ are respectively the unit vectors pointing along the $x$ and the $y$ axes, in this observer's coordinate system. In (24) we have assumed that the horizontal excursions of the electron in $x$ and $y$ are very small 
compared to the Earth's radius. The interaction Hamiltonian for the $\mathbf{h} \cdot \mathbf{h}$ term in (14) is given by

$$
H_{\mathbf{h} \cdot \mathbf{h}}=\frac{m}{2} \mathbf{h} \cdot \mathbf{h}=\frac{m_{e} g^{2} t^{2}}{2 R_{E}^{2}}\left(r^{2} \sin ^{2} \theta\right) .
$$

Therefore, the shift in energy of the atom in the circular Rydberg state, due to the Earth's tidal fields given by (24), is given in first-order perturbation theory by

$$
\Delta E_{\mathbf{h} \cdot \mathbf{h}}=\frac{m_{e} g^{2} t^{2}}{2 R_{E}^{2}} \frac{\left\langle\Psi\left|r^{2} \sin ^{2} \theta\right| \Psi\right\rangle}{\langle\Psi \mid \Psi\rangle} \approx \frac{m_{e} a_{n}^{2}}{2 R_{E}^{2}} g^{2} t^{2}
$$

for large values of $n$, where $n \gg 1$. Once again, since the expectation value in (26) is the mean-square transverse size of the atom, this implies that the size of the atom does not change during free fall, according to first-order perturbation theory. In other words, the atom is "quantum incompressible" in the presence of the inhomogeneous, tidal fields of the Earth, just like in the case of the atom in the presence of an applied DC magnetic field, as long as transitions (i.e., "quantum jumps") out of the initial quantum state $\Psi_{n, n-1, n-1}$ cannot occur. This conclusion is valid assuming that the characteristic frequency of the applied tidal fields is much less than the gap frequency (i.e., the energy gap divided by Planck's constant, which is typically on the order of gigahertz for $n \sim 100$ ) corresponding to a quantum transition from the $n$th state to the nearest adjacent allowed states, and assuming that the tidal gravitational field of the Earth is sufficiently weak.

In the gravitational case, just as in the magnetic case, the energy-level shift caused by the tidal perturbations arising from the Earth's inhomogeneous gravitational field, leads to a force on the atom. This force causes the atom to become a low-field seeker in the inhomogeneous gravitational field of the Earth through the relationship

$$
\left(\mathbf{F}_{\mathbf{h} \cdot \mathbf{h}}\right)_{n}=-\nabla\left(\Delta E_{\mathbf{h} \cdot \mathbf{h}}\right)_{n} \approx-\frac{1}{2} m_{e} a_{n}^{2} t^{2} \nabla\left(\frac{g^{2}}{R_{E}^{2}}\right) .
$$

Thus a hydrogen atom in a circular Rydberg state, which is an extended quantum object, will fall slightly more slowly than a point-like classical test particle which is simultaneously released into free fall along with the atom near the center of mass of the atom in Earth's inhomogeneous field.

The gravitational, Landau-like energy shifts of the atom given by (26) are much too small to be measured directly in Earth-bound experiments using current technology, but in principle they can be measured spectroscopically by monitoring the frequencies of transitions between adjacent Rydberg states, for example, in a satellite laboratory which is in a highly elliptical orbit around the Earth. It is therefore a genuine physical effect.

One might be tempted to say that the force given by (27) questions the universal applicability of the equivalence principle, i.e., the universality of free fall. But it must be kept in mind that the equivalence principle applies strictly to point objects only, inside of which tidal effects can be neglected. This is manifestly not the case for the extended quantum systems being considered here. 


\section{A Superconducting Circuit Consisting of Two Cubes Joined Coherently by Two Parallel Wires}

The analogy between the Rydberg atom and superconducting ring suggests a simple experiment to test the idea of "quantum incompressibility" during free fall, which can be performed in an ordinary laboratory. Consider a horizontal system consisting of two superconducting cubes joined by two parallel superconducting wires to form a superconducting circuit. See Fig. 3.

When a coherent quantum connection between the two cubes is not present (due, say, to the effect of heating coils wrapped around the midsections of both wires which drive them to a normal state by heating them above their transition temperature, so that the coherent quantum connection between the cubes is thereby destroyed), the centers of masses of the two spatially separated cubes, which will have decohered with respect to each other, will follow the converging free-fall trajectories shown in Fig. 1, which are inclined at a slight angle $\theta$ with respect to the vertical plumb line passing through the midpoint "c.m.", with

$$
\theta \approx \frac{L}{R_{E}},
$$

where $L$ is separation of the two cubes, which is also their dimensions (thus chosen for simplicity), and $R_{E}$ is the radius of the Earth. It should be noted that the decoherence, and therefore the spatial separability, of entangled states arising from perturbations due to the environment [6], is a necessary precondition for the applicability of the equivalence principle [7], so that here the universality of free fall can be applied to the free-fall trajectories of the disconnected superconducting cubes. ${ }^{2}$

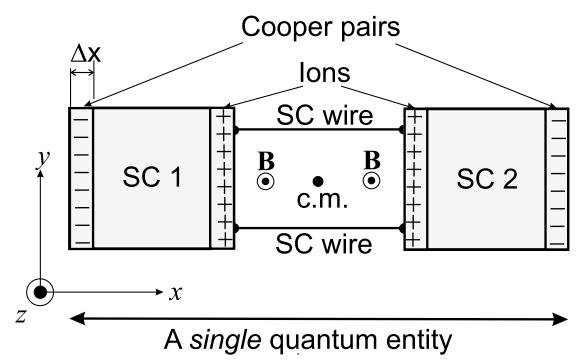

Fig. 3 Two superconducting cubes, SC 1 and SC 2, which are undergoing free fall in Earth's inhomogeneous gravitational field, are connected by means of two thin superconducting wires, which establishes quantum coherence throughout the system, and makes it a single quantum entity with a center of mass ("c.m.") located in the middle. A persistent current through the wires traps a B field inside this superconducting circuit, much like in the circular Rydberg atom. All dimensions of the cubes and the length of the wire are given by the same distance $L$. The $z$ axis denotes the local vertical axis passing through "c.m."

\footnotetext{
${ }^{2}$ The general relativistic concept of a " geodesic" is fundamentally that of a " classical trajectory." As Bohr has taught us, however, the very concept of a "classical trajectory" loses all physical meaning under circumstances in which the uncertainty principle destroys all such classical trajectories. This is the case here for the free-fall trajectories of Cooper pairs inside the superconducting system, since these electron
} 
When a coherent connection is present between the two cubes, they will become a single, macroscopic quantum object like the freely-falling Rydberg atom. The Cooper pairs of electrons of the system will then remain motionless with respect to the midpoint "c.m.", since their macroscopic wavefunction corresponds to a zero-momentum eigenstate relative to c.m., and therefore, by the uncertainty principle, the electrons are completely nonlocalizable within the entire, coherently connected two-cube system. The Cooper pairs of electrons, like the electron in Rydberg atom, will then exhibit quantum incompressibility during free fall. This follows from the fact that the mean-squared size of the coherent electrons of the entire superconducting system remains unchanged in response to the tidal gravitational fields of the Earth, according to first-order perturbation theory.

However, the ions, which have undergone decoherence due to the environment [6, 7], are completely localizable, and therefore, by the equivalence principle, will want to follow the free-fall trajectories that converge onto the center of the Earth shown in Fig. 1. By contrast, the Cooper pairs of electrons will remain coherent during free fall, since they are protected from decoherence by the BCS energy gap [7], and will therefore remain completely nonlocalizable, since they will remain in a zeromomentum eigenstate. This difference in the motion of the ions and of the Cooper pairs of electrons will then lead to the charge-separation effect indicated in Fig. 3, in which the ions will be extruded through the innermost faces of the cubes, because of the convergence of their radial trajectories that point towards the center of the Earth, and in which the Cooper pairs of electrons, which resist this convergence, will be extruded through the outermost faces of the cubes.

The Cooper pairs of electrons in the zero-momentum eigenstate, which remain at rest rigidly with respect to the global c.m. of the entire, coherent two-cube system, will therefore be displaced away from the ions by a distance $\Delta x$ on left face of the left cube, and also on the right face of the right cube. The resulting charge configuration can be approximated by a ball-and-stick model of two charged dumbbells shown in Fig. 4.

On the one hand, the net Coulomb force between the two dumbbells in Fig. 4 is given by

$$
F_{\text {Coulomb }}=\alpha \frac{Q^{2}}{4 \pi \varepsilon_{0} L^{2}},
$$

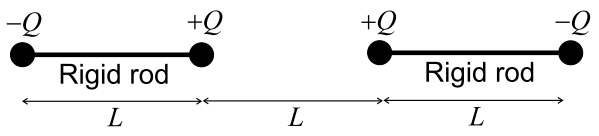

Fig. 4 Ball with charges $-Q$ and $+Q$ are attached to rigid rods with lengths $L$ to form two dumbbells, which model the configuration of charges in Fig. 3. The two innermost charges, both of which are $+Q$, are separated by a distance $L$. These two innermost charges dominate the Coulomb force between the two dumbbells, so that the net force is a repulsive one

pairs will remain in a zero-momentum eigenstate during free fall when they are initially prepared in the BCS ground state at the moment of release into free fall. 
where $\alpha$ is a pure number on the order of unity (this follows from dimensional considerations, since $L$ is the only distance scale in the problem).

On the other hand, the tidal gravitational force between the cubes in Fig. 3 is given by

$$
F_{\text {Tidal }}=M g^{\prime},
$$

where $M$ is the mass of the cube (which is mainly due to the ions), and

$$
g^{\prime}=g \sin \theta \approx g \tan \theta \approx g \theta \approx g L / R_{E}
$$

is the horizontal component of the acceleration due to Earth's gravity acting on the centers of the cubes, which is directed towards the midpoint c.m. of the two cubes. Thus, in equilibrium,

$$
F_{\text {Coulomb }}=F_{\text {Tidal }} .
$$

The voltage difference between the two ends of a given dumbbell (which is a model of the voltage difference between the opposite faces of a given cube) is given by

$$
V=\beta \frac{Q}{4 \pi \varepsilon_{0} L},
$$

where $\beta$ is another pure number of the order of unity (again, this follows from dimensional considerations, since $L$ is only distance scale in the problem). Substituting the squared quantity $Q^{2} / L^{2}$ obtained from (33) into (29), one gets

$$
\begin{aligned}
\alpha \frac{Q^{2}}{4 \pi \varepsilon_{0} L^{2}} & =\alpha \frac{\left(4 \pi \varepsilon_{0}\right)^{2} V^{2}}{\left(4 \pi \varepsilon_{0}\right) \beta^{2}}=4 \pi \varepsilon_{0} \frac{\alpha}{\beta^{2}} V^{2} \\
& =M g^{\prime} \approx \rho L^{3} g \theta \approx \rho g \frac{L^{4}}{R_{E}} .
\end{aligned}
$$

Solving for the voltage difference $V$, one obtains

$$
V \approx\left(\frac{\beta^{2}}{\alpha} \frac{\rho g L^{4}}{4 \pi \varepsilon_{0} R_{E}}\right)^{1 / 2}=\frac{|\beta|}{\sqrt{\alpha}} V_{\mathrm{F}-\mathrm{F}},
$$

where the characteristic free-fall voltage scale $V_{\mathrm{F}-\mathrm{F}}$ for characteristic experimental parameters $\left(L=1 \mathrm{~cm}, \rho=10^{4} \mathrm{~kg} / \mathrm{m}^{3}\right)$ is given by

$$
V_{\mathrm{F}-\mathrm{F}}=\left(\frac{\rho g L^{4}}{4 \pi \varepsilon_{0} R_{E}}\right)^{1 / 2} \sim 1 \text { Volt }
$$

which is experimentally interesting, given the reasonably large capacitances of the two-cube system. (For the geometry of the dumbbells indicated in Fig. 4, the numerical values of $\alpha=11 / 18$ and $\beta=-2 / 3$ are, as indicated earlier, on the order of unity.) 


\section{The "Cavendish-Like" Experiment}

The order-of-magnitude estimate given above indicates that experiments are feasible. An experiment was performed at Merced, in which a slowly time-varying, inhomogeneous, tidal gravitational field is produced by means of two piles of lead bricks placed diametrically opposite each other on a slowly rotating, circular platform, as the sources of the field. The two piles of bricks, which weigh approximately a ton, will orbit slowly and symmetrically around a superconducting circuit similar to the one shown in Fig. 3, which is suspended inside a dilution refrigerator above the center of the rotating platform.

For simplicity, we consider each pendulum to have two support wires, as shown in Fig. 5, so that the motion is constrained along one dimension.

The source masses will rotate around the dilution refrigerator containing the pendula at approximately $1 \mathrm{rpm}$, and so the angular frequency is approximately $\omega=\frac{\pi}{30}$ $s^{-1}$. The gravitational forces on the pendula due to the source masses will thus be periodic in time with a period of approximately 60 seconds (a 30-second period is theoretically possible due to symmetry, but for systematic errors in the setup and design, we will assume 60 seconds). The magnitude of the gravitational forces will vary according to Newton's inverse-square law. In each instant in time, the forces on the pendula can be calculated. Assuming that the angular frequency of the rotating source masses is sufficiently small, a quasi-static model can be implemented, in which the system is in static equilibrium at any given time.

Figure 6 shows the system at an arbitrary instant in time where $\theta=\omega t$. The forces $\mathbf{F}_{1}$ and $\mathbf{F}_{2}$ are gravitational forces exerted by the source masses. $\mathbf{F}_{3}$ is the gravitational force exerted by the opposite pendulum. The separation distance between the two pendula is $s$. Let the vertical faces of the source masses that are (roughly) oriented radially have length $a$, the vertical faces facing the dilution refrigerators have length $b$, and the heights have dimension $c$.

A differential mass element $d M$ of the upper source mass in Fig. 6 exerts a differential force on the right pendulum with magnitude (direction will be considered separately) given by

$$
\left|d \mathbf{F}_{1}\right|=\frac{G m}{x^{2}+y^{2}+z^{2}} d M=\frac{G m \rho}{x^{2}+y^{2}+z^{2}} d V,
$$

Fig. 5 Pendula are supported by two wires each. This configuration causes motion along a single axis, denoted by $x$ in the figure

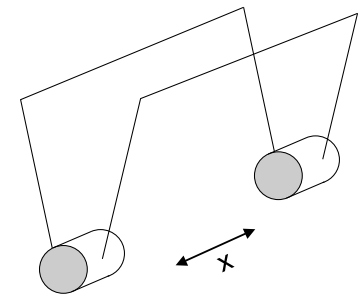


Fig. 6 The experimental setup of the Cavendish-like experiment. The figure shows a snapshot of the dynamic system, where the small displacements of the pendula have, for the moment, been neglected. The coordinates $x, y$ and $z$ will vary with the angle $\theta$, and $\mathbf{i}, \mathbf{j}$ and $\mathbf{k}$ are unit basis vectors

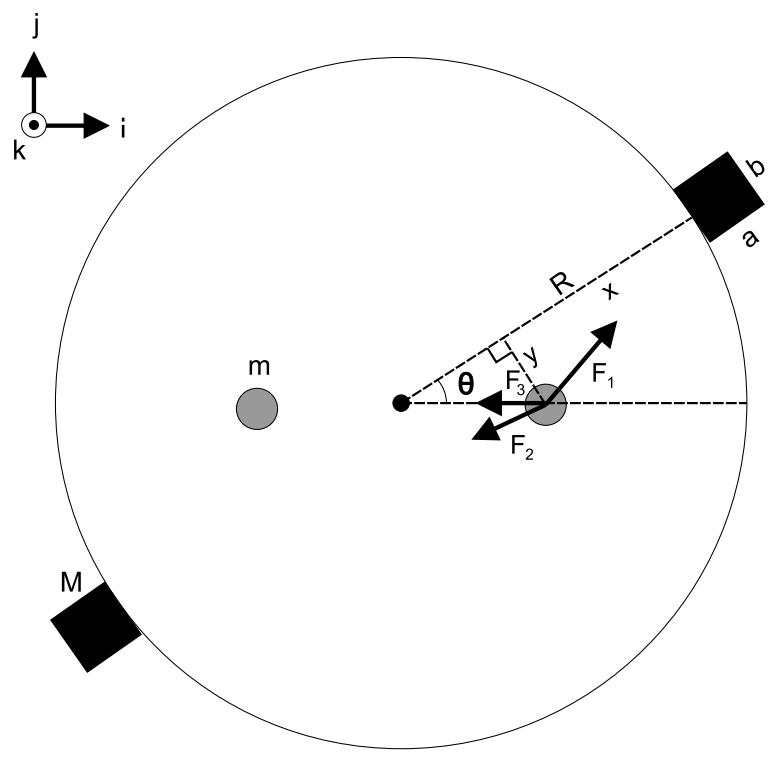

where $\rho$ is the density of the material (assumed to be uniform). Integrating this expression, we obtain the total force exerted by the upper source mass, given by

$$
\left|\mathbf{F}_{1}\right|=G m \rho \int_{-\frac{c}{2}}^{\frac{c}{2}} \int_{\frac{s}{2} \sin \omega t-\frac{b}{2}}^{\frac{s}{2} \sin \omega t+\frac{b}{2}} \int_{R-\frac{s}{2} \cos \omega t}^{R-\frac{s}{2} \cos \omega t+a} \frac{d x d y d z}{x^{2}+y^{2}+z^{2}}
$$

Similarly, the total force exerted on the right pendulum by the lower source mass in Fig. 6 is given by

$$
\left|\mathbf{F}_{2}\right|=G m \rho \int_{-\frac{c}{2}}^{\frac{c}{2}} \int_{\frac{s}{2} \sin \omega t-\frac{b}{2}}^{\frac{s}{2} \sin \omega t+\frac{b}{2}} \int_{R+\frac{s}{2} \cos \omega t}^{R+\frac{s}{2} \cos \omega t+a} \frac{d x d y d z}{x^{2}+y^{2}+z^{2}} .
$$

The angle $\beta$ subtended by vector $\mathbf{F}_{1}$ and the line joining the two pendula in Fig. 6 satisfies the equations

$$
\begin{aligned}
& \cos \beta=\sqrt{\frac{\left(R+\frac{a}{2}\right)^{2} \cos ^{2} \omega t+\frac{s^{2}}{4}-s\left(R+\frac{a}{2}\right) \cos \omega t}{\left(R+\frac{a}{2}\right)^{2}+\frac{s^{2}}{4}-s\left(R+\frac{a}{2}\right) \cos \omega t}} \\
& \sin \beta=\frac{\left(R+\frac{a}{2}\right) \sin \omega t}{\sqrt{\left(R+\frac{a}{2}\right)^{2}+\frac{s^{2}}{4}-s\left(R+\frac{a}{2}\right) \cos \omega t}}
\end{aligned}
$$

and the angle $\gamma$ subtended by $\mathbf{F}_{2}$ and the line joining the two pendula in Fig. 6 satisfies the equations

$$
\cos \gamma=\frac{\frac{s}{2 R+a}+\cos \omega t}{\sqrt{\left(\frac{s}{2 R+a}+\cos \omega t\right)^{2}+\sin ^{2} \omega t}}
$$




$$
\sin \gamma=\frac{\sin \omega t}{\sqrt{\left(\frac{s}{2 R+a}+\cos \omega t\right)+\sin ^{2} \omega t}}
$$

and thus the full expressions for the forces acting on the right pendulum in Fig. 6 are given by

$$
\begin{aligned}
& \mathbf{F}_{1}=G m \rho \int_{-\frac{c}{2}}^{\frac{c}{2}} \int_{\frac{s}{2} \sin \omega t-\frac{b}{2}}^{\frac{s}{2} \sin \omega t+\frac{b}{2}} \int_{R-\frac{s}{2} \cos \omega t}^{R-\frac{s}{2} \cos \omega t+a} \frac{d x d y d z}{x^{2}+y^{2}+z^{2}}(\cos \beta \mathbf{i}+\sin \beta \mathbf{j}), \\
& \mathbf{F}_{2}=-G m \rho \int_{-\frac{c}{2}}^{\frac{c}{2}} \int_{\frac{s}{2} \sin \omega t-\frac{b}{2}}^{\frac{s}{2} \sin \omega t+\frac{b}{2}} \int_{R+\frac{s}{2} \cos \omega t}^{R+\frac{s}{2} \cos \omega t+a} \frac{d x d y d z}{x^{2}+y^{2}+z^{2}}(\cos \gamma \mathbf{i}+\sin \gamma \mathbf{j}),
\end{aligned}
$$

assuming that the pendula and the centers of mass of the source masses are coplanar. Recalling that the pendula are constrained to move along the axis parallel to the unit vector $\mathbf{i}$, the unconstrained components of the forces exerted by the source masses on the right pendulum are given by

$$
\begin{aligned}
& F_{1}=G m \rho \cos \beta \int_{-\frac{c}{2}}^{\frac{c}{2}} \int_{\frac{s}{2} \sin \omega t-\frac{b}{2}}^{\frac{s}{2} \sin \omega t+\frac{b}{2}} \int_{R-\frac{s}{2} \cos \omega t}^{R-\frac{s}{2} \cos \omega t+a} \frac{d x d y d z}{x^{2}+y^{2}+z^{2}} \\
& F_{2}=G m \rho \cos \gamma \int_{-\frac{c}{2}}^{\frac{c}{2}} \int_{\frac{s}{2} \sin \omega t-\frac{b}{2}}^{\frac{s}{2} \sin \omega t+\frac{b}{2}} \int_{R+\frac{s}{2} \cos \omega t}^{R+\frac{s}{2} \cos \omega t+a} \frac{d x d y d z}{x^{2}+y^{2}+z^{2}}
\end{aligned}
$$

The force exerted by the left pendulum on the right pendulum is anti-parallel to $\mathbf{i}$, and its magnitude is given by

$$
F_{3}=\frac{G m^{2}}{s^{2}} .
$$

Viewing the right pendulum from the side, the free body diagram can be pictured as in Fig. 7.

Fig. 7 Force body diagram of single pendulum

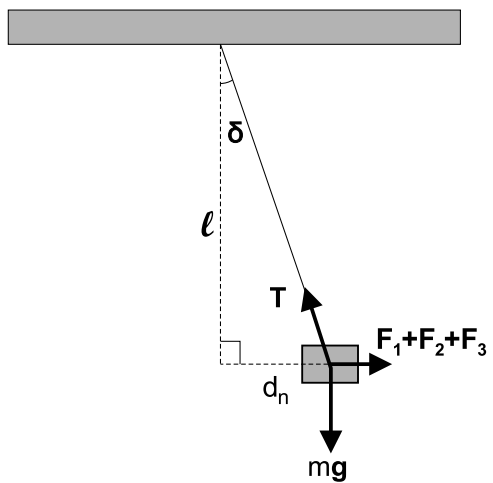


Comparing the vertical and horizontal components of the vectors in Fig. 7, we obtain an expression for the deflection magnitude $d_{n}$, given by

$$
d_{n} \approx \frac{\ell}{m g}\left(F_{1}-F_{2}-F_{3}\right),
$$

where we have assumed $d_{n}$ to be small, so that (47) is explicit. The subscript $n$ is used for the case that the pendula are normal, so that both the ionic lattice and the valence electron system follow the same local geodesics. $F_{3}$ can be safely neglected if the source masses are sufficiently large.

Let us now turn to the concept of charge separation within superconductors. Let the pendula now be superconducting, and share a continuous superconducting connection, so that the Cooper-pair wavefunction has a constant phase across both pendula. If we assume that only the ionic lattice of the pendula are subject to the gravitational forces of the source masses, a charge separation will ensue. For the moment, let us assume that the Cooper pair wavefunction is completely rigid, and that the superfluid will therefore remain motionless with respect to the center of mass of the pendulum system, which lies approximately at the midpoint between the two pendula. This charge separation will cause Coulomb forces both between the two pendula, and within the pendula themselves. These forces must be taken into account to accurately predict the motions of the ionic lattices, and the magnitude of the charge separation.

Figure 8 shows the pendula at an instant in time when the ionic lattices are pulled outwards by the source masses. The charged faces of the pendula are modeled here using point charges.

Summing the Coulombic forces that the left pendulum exerts on the ionic lattice of the right pendulum in Fig. 8, it can be shown that the total Coulombic force is

$$
\mathbf{F}_{Q, d>0} \approx \frac{-Q^{2}}{4 \pi \epsilon_{0} s^{2}}\left[\frac{L(2 s+L)}{(s+L)^{2}}\right] \mathbf{i},
$$

where we have ignored the charge extrusion distance $d$, since $d$ is much smaller than $s$ and $L$. The Coulomb force on the ionic lattice of the right pendulum exerted by the electron superfluid within the right pendulum, due to charge separation, is

$$
\mathbf{F}_{c, d>0} \approx-\frac{Q^{2}}{4 \pi \epsilon_{0} L^{2}} \mathbf{i}
$$

where the same approximation has been used. The subscript $d>0$ has been used since (48) and (49) are only valid when the positively-charged ionic lattice is extruded

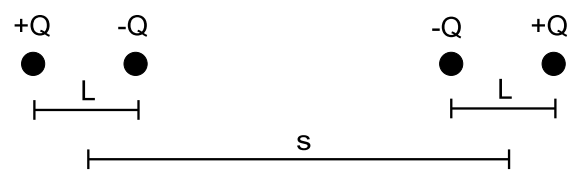

Fig. 8 Superconducting pendula under the influence of tidal forces that produce charge separation. The physical dimension of the pendulum along the axis of the charge separation is given by $L$, and the center-to-center distance between the pendula is given by $s$ 
from the negatively-charged superfluid on the outer faces of the pendula. When the signs of the charges are reversed, the forces on the ionic lattice of the right pendulum can be found by making the substitution $L \rightarrow-L$, so the general expression for the Coulombic forces on the right pendulum are

$$
\mathbf{F}_{Q} \approx \frac{-Q^{2}}{4 \pi \epsilon_{0} s^{2}}\left\{\frac{L \operatorname{sgn}(d)[2 s+L \operatorname{sgn}(d)]}{[s+L \operatorname{sgn}(d)]^{2}}\right\} \mathbf{i}
$$

and

$$
\mathbf{F}_{c} \approx-\frac{Q^{2} \operatorname{sgn}(d)}{4 \pi \epsilon_{0} L^{2}} \mathbf{i}
$$

where

$$
\operatorname{sgn}(d)= \begin{cases}1, & d>0 \\ 0, & d=0 \\ -1, & d<0 .\end{cases}
$$

Considering all forces on a single superconducting pendulum, we have a model that is similar to that depicted in Fig. 7, but $\mathbf{F}_{Q}$ and $\mathbf{F}_{c}$ are considered, in addition to $\sum_{i=1}^{3} \mathbf{F}_{i}$. For this case, we have

$$
d \approx \frac{\ell}{m g}\left(F_{1}-F_{2}-F_{3}-F_{Q}-F_{c}\right)
$$

where $F_{Q}$ and $F_{c}$ are the magnitudes of the vectors defined in (50) and (51), respectively.

The extruded charge $Q$ depends on the extrusion length. Starting with

$$
Q=2 e n_{s} V
$$

where $n_{s}$ is the superconducting electron density, $-2 e$ is the Cooper pair charge, and $V$ is the extrusion volume. The current experimental design involves cylindricallyshaped pendula, so (54) becomes

$$
Q=2 e n_{s} \pi r^{2} d
$$

where $r$ is the cylindrical radius of the sample. Thus,

$$
d=\frac{Q}{2 e n_{s} \pi r^{2}},
$$

and it should be noted here that

$$
\operatorname{sgn}(d)=\operatorname{sgn}(Q)
$$

Substitution of (56) and (57) into (53), we have 
Fig. 9 Predicted charge signal as a function of time

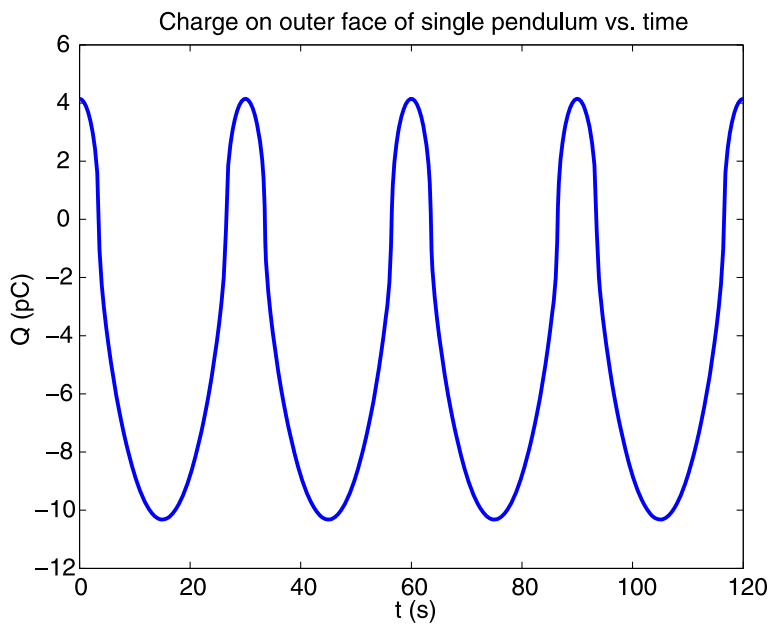

$$
\begin{aligned}
Q= & \frac{2 e n_{s} \pi r^{2} \ell}{m g}\left(F_{1}-F_{2}-F_{3}-\frac{Q^{2} \operatorname{sgn}(Q)}{4 \pi \epsilon_{0} s^{2}}\left\{\frac{L[2 s+L \operatorname{sgn}(Q)]}{[s+L \operatorname{sgn}(Q)]^{2}}\right\}\right. \\
& \left.-\frac{Q^{2} \operatorname{sgn}(Q)}{4 \pi \epsilon_{0} L^{2}}\right) .
\end{aligned}
$$

An iterative numerical method was used to solve (58) for $Q$. It should also be noted that the expressions for $F_{1}$ and $F_{2}$ were evaluated using numerical integration. A plot of the expected charge as a function of time using actual experimental parameters is depicted in Fig. 9.

We expect to be able to see (using synchronous detection) the charge separation induced by these gravitational fields in a superconducting circuit, which consists of two well-separated superconducting bodies, both of which are suspended by means of pairs of superconducting wires inside the same refrigerator, so that the two bodies form the superconducting plumb bobs of two pendula. These two bodies are then coherently connected to each other by means of a pair of parallel superconducting wires, as indicated in Fig. 3, to form a single superconducting circuit, i.e., a single quantum entity. The charge separation effect can then be measured inductively by means of a sensitive electrometer (we should be able to see the charge induced by the extruded Cooper pairs, which should be on the order of picocoulombs, with a high signal-to-noise ratio). If we should observe a nonzero charge-separation signal in this experiment, then this observation would establish the existence of a novel coupling between gravity and electricity mediated by means of macroscopic quantum matter.

Normally, the gravitational fields due to the lead bricks should cause small angular deflections on the order of nanoradians of the two pendula. These small deflections should occur relative to the local vertical axis which is located at the midpoint in between them (see Fig. 3, in which the two freely-falling objects are replaced by the two plumb bobs of the two pendula). We would normally expect to see such deflections if these two pendula consisted of normal, classical matter, or if they consist of two superconducting plumb bobs which have had their superconducting connection between them destroyed due to decoherence. Such deflections could be measured with 
high signal-to-noise ratios using laser interferometry. If we were to monitor both the deflections of the pendula and the charge separation effect in the same experiment, there would be four logical possibilities as to the possible outcomes:

(I) Charge-separation? YES. Deflection? NO.

(II) Charge-separation? NO. Deflection? YES.

(III) Charge-separation? YES. Deflection? YES.

(IV) Charge-separation? NO. Deflection? NO.

Based on the arguments presented above, we would expect (I) to be the outcome, if the Cooper pairs were to be able to drag the ions of the lattice into co-motion with these superconducting electrons during free fall. In the tug-of-war between the uncertainty principle and the equivalence principle, the uncertainty principle wins in (I). By contrast, if there is nothing special about this superconducting system over any other material system, i.e., if the universality of free fall were to apply to the Cooper pairs inside the superconducting circuit so that they would undergo free fall along with the ions, and therefore the superconducting system would remain electrically neutral and unpolarized during free fall, then we would expect (II) to be the outcome. The equivalence principle wins in (II). If, however, there does exist a charge-separation effect, but the ions of the lattice were to drag the Cooper pairs into co-motion with the ionic lattice during free fall, then we would expect (III) to be the outcome. Finally, there exists the remote possibility of outcome (IV), which would indicate that Newtonian gravity would somehow have failed to produce any deflection at all of the pendula in this experiment, but that nevertheless the system remains electrically neutral and unpolarized in the presence of the ton of bricks. Results from this "Cavendish-like" experiment will be presented elsewhere.

Under the present experimental conditions of the Cavendish-like experiment, two of the four outcomes listed above have been ruled out. To understand why let us consider one of the superconducting pendula plumb bobs, and suppose that a stable DC charge separation effect exist within the plumb bob for $t \leq 0$. Note that, as shown above, this DC charge separation effect would lead to an internal voltage drop of approximately 1 Volt across the superconducting plumb bob. By stable, it is meant that there are no time changing quantities so that

$$
\frac{\partial \Phi_{B}}{\partial t}=0, \quad \frac{\partial A}{\partial t}=0 \quad \text { and } \quad \frac{\partial \Phi_{E}}{\partial t}=0 ; \quad t \leq 0 .
$$

Because there is no external electric field to cancel out the internal electric field produced by the predicted stable DC charge separation effect for $t \leq 0$, there must be a measurable voltage drop across the superconducting plumb bob. This is to be contrasted with the case of a conductor placed between two parallel plates of a capacitor where there exists a stable charge separation effect but no measurable voltage drop across it. Hence, if we allow the system to evolve at $t>0$, the Cooper pairs deep inside the SC will experience a scalar potential $(\phi)$ which imparts energy to the pairs.

$$
|E|=q \phi=2 e \phi \sim 2 \mathrm{eV},
$$

where we set $\phi=1$ Volt as predicted above. Comparing this energy with the ground state energy gap involved in most conventional type I superconductors, $E_{g} \sim \mathrm{meV}$, 
we see that such scalar potential would excite the Cooper pairs into the quasi-particle state, thus leading to a pair-breaking mechanism. Therefore, such DC charge separation effect would not be stable and there would be no measurable charge separation in the present Cavendish-Like experiment. Hence outcomes (I) and (III), where a measurable stable DC charge separation effect are expected, have already been ruled out, leaving us with outcome (II) and (IV) as the only remaining two possibilities, though in an experiment involving high-frequency perturbations, these two outcome possibilities may remain.

Acknowledgements The authors thank Prof. Michael Scheibner, Bong-Soo Kang, and Philip Jensen for their assistance concerning the Cavendish-like experiment.

Raymond Chiao wishes to extend his heartiest birthday congratulations to Danny and Helmut!

Open Access This article is distributed under the terms of the Creative Commons Attribution Noncommercial License which permits any noncommercial use, distribution, and reproduction in any medium, provided the original author(s) and source are credited.

\section{References}

1. Hulet, R., Kleppner, D.: Phys. Rev. Lett. 51, 1430 (1983)

2. Haroche, S., Raimond, J.-M.: Exploring the Quantum. Oxford University Press, London (2006)

3. DeWitt, B.S.: Phys. Rev. Lett. 16, 1092 (1966)

4. Landau, L.D., Lifshitz, E.M.: Quantum Mechanics. Butterworth-Heinemann, Oxford (2003)

5. Minter, S.J., On the implications of incompressibility of the quantum mechanical wavefunction in the presence of tidal gravitational fields. Ph.D. diss., University of California (2010)

6. Zurek, W.H.: Rev. Mod. Phys. 75, 715 (2003)

7. Minter, S.J., Wegter-McNelly, K., Chiao, R.Y.: Physica E 42, 234 (2010)

8. Parker, L.: Phys. Rev. D 22(8), 1980 (1922)

9. Parker, L.: Phys. Rev. Lett. 44, 1559 (1980)

10. Parker, L.: Phys. Rev. D 25(12), 1982 (1922)

11. Gill, E., et al.: Class. Quantum Gravity 4, 1031 (1987) 